\title{
Cellule de parachèvement pour pièce de fonderie de grandes dimensions
}

\author{
Laurent Sabourin $^{1, a}$, Vincent Robin ${ }^{1}$, Grigore Gogu ${ }^{1}$ et Jean-Michel Fauconnier ${ }^{2}$ \\ ${ }^{1}$ Laboratoire de Mécanique et Ingénieries, Institut Français de Mécanique Avancée (IFMA), Université Blaise Pascal \\ (UBP), Les Cézeaux, rue Roche Genès, BP 265, 63175 Aubière Cedex (EA 3867, FR TIMS/CNRS 2856), France \\ 2 ALCAN, ZI Le Piat, 63502 Issoire, France
}

Reçu le 10 décembre 2010, accepté le 10 octobre 2011

\begin{abstract}
Résumé - L'obtention de pièces structurales en fonderie sable aluminium de haute technicité nécessite un processus de parachèvement conséquent comprenant la découpe du système de coulée, suivie d'un ponçage quasi global des surfaces. Les exigences industrielles en termes de sécurité et de rentabilité remettent fortement en cause la réalisation manuelle de ces opérations. Cet article présente le développement d'une nouvelle cellule robotisée pour ce parachèvement, mené en collaboration avec La Société des Fonderies d'Ussel (SFU) du groupe ALCAN. La première partie est consacrée à la caractérisation des contraintes liées aux spécifications fonctionnelles attendues sur la pièce et aux procédés d'usinage et de ponçage. Ceci a abouti à la définition d'une cellule redondante 8 axes pour répondre au critère d'espace de travail et intégrant un robot à structure hybride (Tricept) pour ses capacités à réaliser les opérations d'usinage en conditions UGV et de ponçage. La deuxième partie porte sur la définition de critères pour une gestion optimisée des redondances en vue d'améliorer le comportement global de la cellule et répondre aux exigences de précision et de capacité cinématique. La dernière partie présente l'optimisation mise en œuvre et les résultats validés sur un ensemble de pièces.
\end{abstract}

Mots clés : Robot hybride / fraisage / ponçage / redondances cinématiques / contraintes des procédés / optimisation multi-objectifs

\begin{abstract}
Robotized cell dedicated to finishing operations, by machining and polishing, on large cast parts. The production of structural parts in a highly-specialized aluminium foundry requires a substantial finishing process, including the machining of the casting system followed by the polishing of all the surfaces. Industrial requirements, in terms of economic and safety factors, call into question the manual performance of finishing operations. This paper presents the development of a new robotized cell dedicated to finishing operations, carried out with the Société des Fonderies d'Ussel (SFU), part of the ALCAN group. We first formalize the constraints related to functional requirements and the machining and polishing process. The architecture is kinematically redundant and contains 8 controlled axes to fulfill the workspace criterion. The hybrid structure (Tricept) is integrated for its kinematic capability to perform machining and polishing processes in HSM conditions. The second part relates to the definition of criteria to optimize kinematic redundancy in order to improve accuracy requirements and kinematic capacity. The final section is dedicated to optimization; the results were validated on industrial parts.
\end{abstract}

Key words: Parallel robot / milling / polishing / kinematics redundancy / process constraints / multiobjective optimization

\section{Introduction}

La réalisation de pièces structurales à faibles épaisseurs et de grandes dimensions (jusqu'à 1,2 × $3 \mathrm{~m}$ pour les pièces considérées) en fonderie sable aluminium nécessite des moules complexes et le procédé génère

\footnotetext{
${ }^{a}$ Auteur pour correspondance: sabourin@ifma.fr
}

des déformations sur la pièce difficilement prévisibles. L'obtention de la pièce finie nécessite une opération de parachèvement conséquente comprenant d'une part la découpe du système de coulée, déjà en partie robotisée, et d'autre part l'élimination des attaques de coulées, bavures, etc., suivies d'un ponçage quasi global des surfaces, réalisé manuellement. Or, les exigences industrielles en 
termes de sécurité et de rentabilité imposent l'automatisation de celles-ci. La nouvelle cellule robotisée doit permettre avec un même effecteur la réalisation d'enlèvement de matière par coupe et abrasion, ainsi que le recalage des trajectoires pour s'adapter aux pièces réelles déformées. Le couplage de ces procédés sur un même effecteur, encore marginal, trouve de plus en plus de débouchés. De nombreux travaux sont menés sur le polissage robotisé autour de la compréhension du phénomène d'abrasion $[1,2]$ et la génération des trajectoires [3,4] et d'autres se développent également sur machine-outil [5]. De même, l'essor de l'Usinage Grande Vitesse (UGV) a vu le développement de robots et machines-outils à architectures parallèles et hybrides [6-9] pour leurs performances dynamiques supérieures aux architectures sérielles. Des solutions de couplage des procédés ont également été proposées [10] avec une solution intéressante du robot hybride RNT [11]. Dans le cadre des travaux présentés, le couplage robotisé des procédés sur un même effecteur nécessite un compromis entre capacités cinématiques, rigidité, dextérité et espace de travail.

Dans la première partie, cet article présente l'étude préliminaire de l'ensemble des contraintes produit inhérentes à l'obtention des pièces par fonderie, des procédés d'usinage et de ponçage vis-à-vis des capacités attendues de la cellule. Ceci a abouti à une cellule redondante ( 8 ddls) intégrant un robot de type Tricept et deux axes externes, dont le comportement est non isotrope dans l'espace de travail. La deuxième partie présente la démarche mise en œuvre dans la gestion optimisée de la redondance pour l'amélioration des capacités de précision et cinématique. Après avoir précisé les critères d'optimisation, en regard des différentes contraintes, nous présentons les résultats de l'optimisation multicritères à poids variables et la mise en application sur des pièces industrielles.

\section{Contraintes des procédés}

\subsection{Intérêts et contraintes du procédé de fonderie}

Le procédé de fonderie sable permet de réaliser des pièces de grandes dimensions et de formes complexes intégrant un grand nombre de fonctions : structure générale, reprise d'efforts, canaux de passages de fluides, surfaces d'assemblage, surfaces d'échange, cosmétique. En raison de la complexité des pièces et des contraintes de poids (pièces aéronautiques en particulier), les pièces présentent de faibles épaisseurs et sont fortement nervurées. Les contraintes métallurgiques de coulée imposent une alimentation multiple en alliage qui se traduit par la présence de très nombreuses attaques de coulées (Fig. 1).

Le moulage sable induit également une variabilité dimensionnelle et les pièces structurales à faibles épaisseurs présentent des déformations difficilement prévisibles : ouverture, torsion. Dans le cadre de cette activité, l'obtention de la pièce finie nécessite une opération de parachèvement conséquente comprenant d'une part l'élimination des attaques de coulée, des bavures et des marques
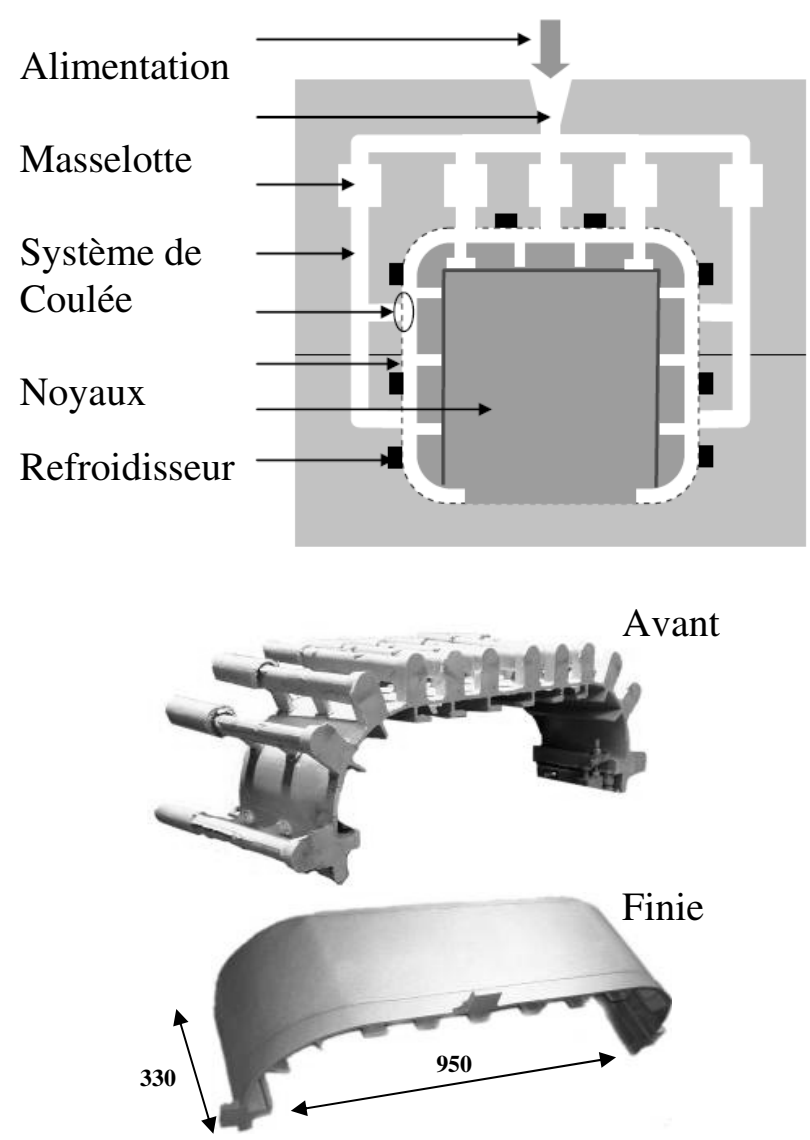

Fig. 1. Caractéristiques du procédé de fonderie sable de haute technicité.

Pièce

Pièce réelle

Usinage des attaques de coulée

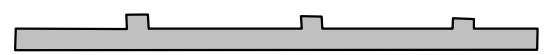

Ponçage

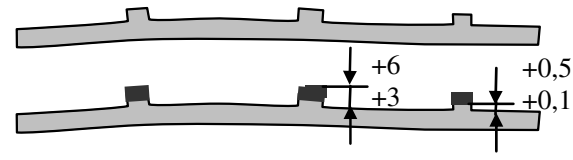

Fig. 2. Parachèvement des pièces.

de refroidisseur et d'autre un ponçage global de la couche superficielle d'alumine (Fig. 2). Cette opération est suivie ou non d'un sablage ou d'un grenaillage qui permet de garantir les caractéristiques mécaniques par traitement de précontraintes superficielles.

Une contrainte forte est le respect du profil réel, l'usinage des attaques de coulée ne doit en aucun cas venir entailler la pièce et le ponçage doit respecter le profil réel. En raison de la variabilité dimensionnelle et de l'accessibilité parfois difficile aux zones de travail, l'ensemble des opérations de parachèvement (burinage et ponçage) est encore réalisé manuellement, ce qui génère également une variabilité en termes de qualité d'autant plus sensible sur les aspects cosmétiques. Les exigences fonctionnelles attendues sur les pièces concernent : la tolérance du parachèvement vis-à-vis de la pièce (défaut de forme 

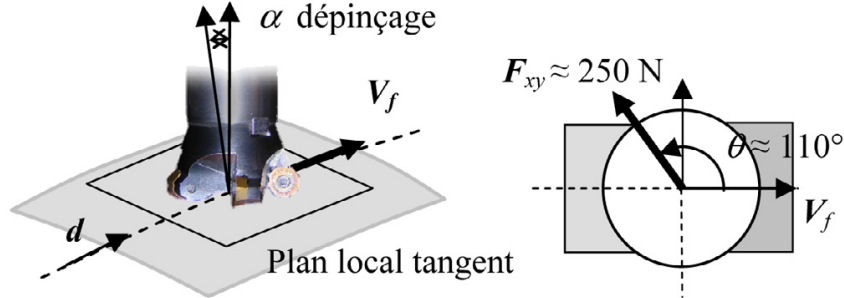

Fig. 3. Stratégie en usinage et composante des efforts.

$<0,4 \mathrm{~mm}$ pour des surfaces particulières et $0,8 \mathrm{~mm}$ avec positionnement), l'état de surface final désiré $(\leq 6,3 \mu \mathrm{m}$ pour les fonctions cosmétiques) et le respect de l'intégrité des surfaces réelles (Fig. 2).

SFU dispose déjà de deux cellules robotisées d'usinage intégrant des robots Tricept qui réalisent, sur un ensemble de pièces, les étapes de dégrappage et d'ébarbage. Elles montrent cependant leurs limites en particulier par leur manque de flexibilité (programmation par apprentissage, problèmes du recalage des trajectoires) et leur incapacité à réaliser des opérations de ponçage. Par rapport aux critères tels que la taille des pièces, la complexité d'accessibilité, la variabilité et l'état déformé des pièces, et fort de l'expérience des précédentes cellules, SFU a écarté l'intégration d'une machine-outil, notamment en terme de coût et de développement de nouvelles compétences. Il s'agit donc de caractériser les contraintes fortes des procédés sur les caractéristiques attendues de la cellule robotisée.

\subsection{Contraintes en usinage}

Pour des critères économiques, le choix de l'usinage s'est porté vers l'utilisation de conditions UGV qui nécessite une étude précise du couple outil-matière. Les essais de réglage ont été réalisés sur machine UGV Météor10 équipée d'une table Kissler permettant de valider les paramètres de la stratégie de balayage 5 axes (Fig. 3) : usinage en avalant avec dépinçage $\left(\alpha=3^{\circ}\right)$ en Zig suivant la direction $\boldsymbol{d}$, fraise carbure 5 dents $\varnothing 63 \mathrm{~mm}$ $\left(n=12000\right.$ tr. $\left.\mathrm{min}^{-1}, \boldsymbol{V}_{f}=6000 \mathrm{~mm} \cdot \mathrm{min}^{-1}, a_{p}=3 \mathrm{~mm}\right)$ pour une puissance consommée fixée à $10 \mathrm{~kW}$ maxi à la broche, et les sollicitations en efforts [12]. Les passes sont réalisées centrées par rapport aux plots d'une largeur moyenne de $40 \mathrm{~mm}$. Le trajet en Zig permet de conserver les mêmes sollicitations entre chaque passe.

Parmi l'ensemble des paramètres, un des facteurs prépondérants pour garantir la qualité de coupe en UGV est le respect de la vitesse d'avance $\boldsymbol{V}_{f}$ constante [13] suivant $\boldsymbol{d}$, qui est directement en relation avec le taux d'enlèvement de matière dépendant directement de l'avance à la dent $f_{z}$ définie par :

$$
f_{z}=\frac{\boldsymbol{V}_{f}}{z \cdot n}
$$

où $n$ et $z$ sont respectivement la fréquence de rotation et le nombre de dents. L'enlèvement de matière est matérialisé
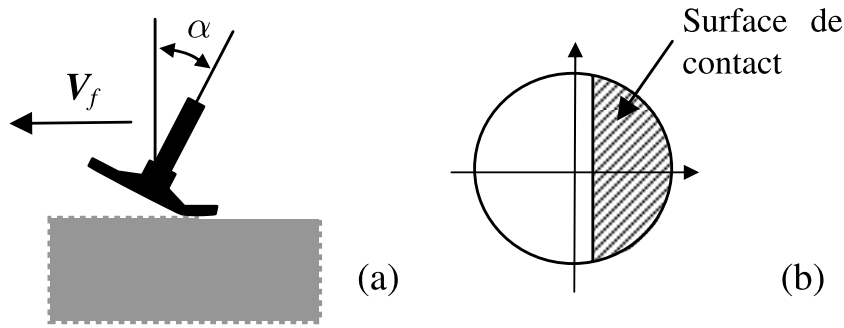

Fig. 4. Stratégie (a) et modélisation de la surface active du disque en ponçage (b).

par l'enveloppe du trajet des arêtes de coupe. La diminution de la vitesse d'avance $\boldsymbol{V}_{f}$ entraîne une diminution de l'avance à la dent $f_{z}$. Ceci ne modifie pas l'enveloppe d'enlèvement de matière mais peut provoquer une détérioration de la qualité de coupe. Cela se traduit par une usure prématurée, un mode vibratoire ou encore une dégradation de l'état de surface.

\subsection{Contraintes en abrasion}

La stratégie retenue pour le ponçage est de type balayage 5 axes en Zig avec disques souples $\varnothing 115 \mathrm{~mm}$, dépinçage $\left(\alpha=5^{\circ}\right)$ et les paramètres suivants $(n=$ 12000 tr. $\min ^{-1}, V_{f}=2400 \mathrm{~mm} \cdot \mathrm{min}^{-1}$ ) (Fig. 4a). L'enlèvement de matière est assuré par le contrôle de l'effort de contact entre l'outil et la pièce au moyen d'un système compliant mécanique piloté par servovalve (Mape) intercalé entre la broche et l'effecteur [14] qui adapte la trajectoire au profil réel de la pièce. Le taux d'enlèvement de matière est donné par la relation de Preston :

$$
\frac{\mathrm{d} h}{\mathrm{~d} t}=k_{p} \cdot P_{c} \cdot V_{r}
$$

où $h$ est la hauteur de matière enlevée, $P_{c}$ la pression de contact locale, $V_{r}$ la vitesse relative entre la pièce et l'abrasif, et $k_{p}$ le coefficient dit de Preston. La taille du grain, le taux d'abrasif, le couple des matériaux ainsi que les différents phénomènes liés à l'enlèvement de matière ne sont pas pris en compte séparément mais regroupés dans le coefficient de Preston. Ceci nécessite de déterminer expérimentalement, pour chaque condition d'utilisation, le coefficient correspondant [15].

Une première approximation de l'épaisseur enlevée sur une zone est donnée par l'intégrale sur le temps d'appui sur la zone (Fig. 4b) :

$$
e=\int_{\text {temps }} k_{\text {d'appui }} \cdot P_{c} \cdot V_{r} \cdot \mathrm{d} t
$$

À conditions de travail constantes (effort d'appui, vitesse de rotation, etc.), l'enlèvement de matière est donc directement proportionnel au temps passé, donc à l'inverse de la vitesse d'avance. La diminution de la vitesse d'avance $\boldsymbol{V}_{f}$ entraîne alors une augmentation de l'enlèvement de matière qui se traduit par un creusement de la surface. Nous devons donc garantir un taux d'enlèvement de 
matière constant au cours du temps. La direction de balayage $\boldsymbol{d}$ quant à elle est définie en fonction des accessibilités aux surfaces et minimisant le nombre de trajectoires d'entrée et de sortie matière toujours délicate en ponçage, ou optimisée par un couplage étroit en usinage et ponçage pour les pièces spécifiques intégrant des contraintes cosmétiques particulières.

\subsection{Couplage des procédés}

L'étude préalable de faisabilité a permis de définir la tolérance de surépaisseur d'usinage admissible sur les plots $(+0,1,+0,5 \mathrm{~mm})$ pouvant être éliminée par l'opération de ponçage (Fig. 2). L'usinage de chaque plot nécessite au préalable un recalage local (position et orientation) afin de garantir l'intégrité de la pièce et d'affleurer au mieux la surface réelle.

\subsection{Bilan et traduction des contraintes}

Dans les deux cas (usinage et ponçage), la première contrainte est de garantir à l'effecteur une vitesse de déplacement constante égale à la vitesse d'avance $V_{f}$ imposée par la stratégie. Les contraintes supplémentaires imposées à l'effecteur concernent : le suivi de trajectoires continues (sans reconfiguration), la prise en compte de la charge embarquée et les efforts de coupe. L'ensemble des contraintes se répercute sur la cellule robotisée en termes de :

- précision de la tâche $\rightarrow$ précision du porteur;

- précision de la tâche + charge et efforts de coupe $\rightarrow$ rigidité du porteur;

- enveloppe de travail et accessibilité $\rightarrow$ grand espace de travail ;

- Trajectoires 5 axes et continues $\rightarrow$ génération de trajectoires parcourables;

- vitesses d'avance $V_{f}$ élevées et constantes $\rightarrow$ admissibilité cinématique.

\section{Définition de la cellule}

La nécessité d'intégrer un capteur extéroceptif de vision pour le recalage des trajectoires et un contrôle en effort pour le ponçage ne sont pas des fonctionnalités standard d'une machine-outil. Le choix d'un robot à architecture hybride Tricept a été retenu mais il comporte un espace de travail réduit. Celui-ci est étendu par l'ajout de deux mobilités cinématiques conférant à l'ensemble un volume cylindrique de $\varnothing 1,5 \mathrm{~m} \times 3 \mathrm{~m}$.

L'architecture finale retenue comprend : un robot Tricept ABB IRB 940 (6 axes), déplacé par un axe linéaire (track) et associé à un axe rotatif (retourneur) portant la pièce (Fig. 5a). La cellule ainsi constituée comporte 8 axes asservis (Fig. 5b) pilotés par une baie ABB S4C+. Cette structure est complétée par deux broches : $12 \mathrm{~kW}$ pour l'usinage, $8 \mathrm{~kW}$ pour le ponçage montées sur une

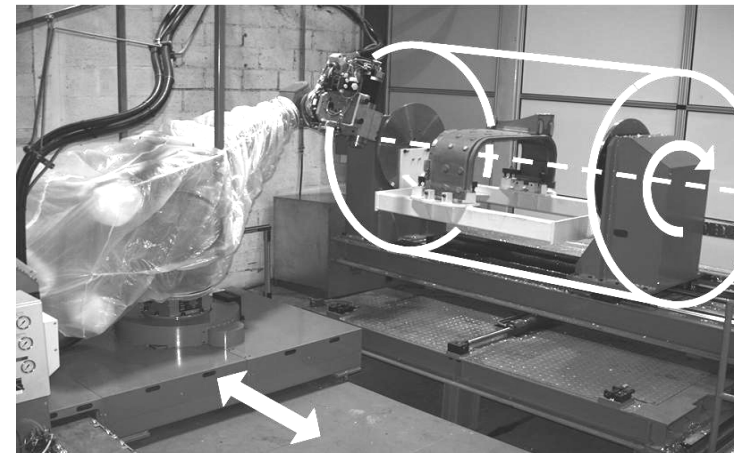

(a)

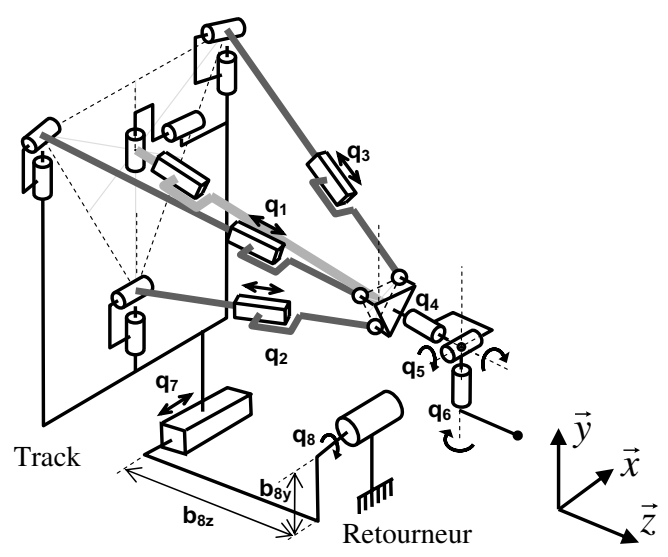

(b)

Fig. 5. Architecture de la cellule (a) et schéma cinématique correspondant (b).

Tableau 1. Influence des facteurs.

\begin{tabular}{cc}
\hline Exactitude & Répétabilité \\
\hline Modélisation & Commande \\
Poids propre & Asservissement \\
Effets thermiques & Modes propres \\
& Sollicitations de la tâche \\
\hline
\end{tabular}

compliance passive pilotée, un système d'acquisition et de traitement d'image 3D (VisioNerf) pour le recalage des trajectoires, l'ensemble étant associé à un changeur de tête et un magasin d'outils.

Cependant, le comportement réel du système dans l'espace de travail est fortement anisotrope [16] et nous le caractérisons par l'introduction de trois notions :

- Précision de la cellule : la précision du trajet est associée à deux grandeurs (Tab. 1), l'exactitude et la répétitivité, dont l'étendue est liée à une multitude de facteurs [17]. Les valeurs annoncées pour le robot IRB 940 sont de 0,2 $\mathrm{mm}$ en exactitude et de 0,02 $\mathrm{mm}$ en répétitivité soit un facteur 10 entre ces deux grandeurs.

Concernant l'exactitude, répétable, celle-ci est en partie compensée par le recalage par vision en prenant soin de conserver la même configuration du robot lors de la phase d'acquisition vision et d'usinage des plots et une définition de repères outils analogues. Cependant, ces deux grandeurs sont également affectées par les déformations de la structure, liées d'une part 

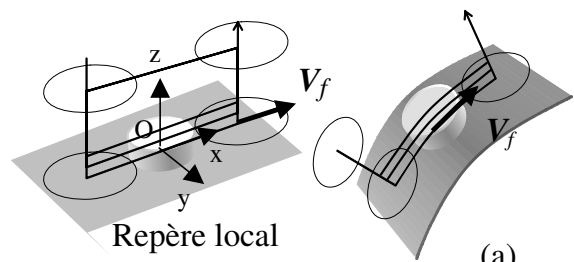

(a)

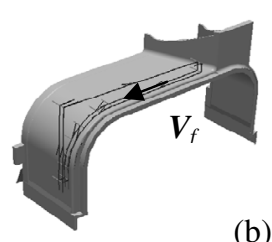

(b)

Fig. 6. Trajets outil en usinage (a) et en ponçage (b).

aux sollicitations externes et d'autre part à la rigidité de celle-ci, elle-même liée à la configuration articulaire instantanée. L'objectif est de minimiser les déformations en utilisant la redondance cinématique pour modifier la configuration articulaire.

- Admissibilité cinématique : une configuration de la cellule, pour une pose donnée, sera dite cinématiquement admissible si elle permet de satisfaire la contrainte de vitesse d'avance $\boldsymbol{V}_{f}$ constante imposée par la stratégie. Ceci dépend des limites des vitesses articulaires du robot et de sa configuration instantanée.

- Parcourabilité des trajectoires : la notion de tparcourabilité désigne la capacité à suivre une trajectoire de manière continue [18]. Dans le cas des environnements sans obstacle, une trajectoire qui ne traverse pas une frontière d'aspect peut être intégralement parcourue de manière continue par l'effecteur. Ceci impose que les valeurs articulaires calculées pour l'ensemble des poses ne franchissent pas de singularité.

Ces deux dernières dépendent également de la définition des trajectoires et du positionnement de la pièce.

\section{Définition des trajectoires}

Les parcours outils sont définis par des segments de droite et arcs de cercle raccordés en tangence à partir du modèle numérique de la pièce sous CatiaV $5{ }^{\circledR}$. Les trajectoires, générées dans RobotStudio ${ }^{\circledR}$, sont définies en minimisant le nombre de poses (Fig. 6). La définition d'une tolérance de la zone de raccordement des trajets autour de chaque pose permet d'utiliser au mieux les fonctionnalités de la commande $\mathrm{ABB}$ en assurant une grande régularité de la vitesse de déplacement de l'effecteur et limitant ainsi les sollicitations dynamiques sur la structure.

En usinage, chaque trajectoire est associée à un repère local défini au centre des plots (Fig. 6a) ajusté ensuite sur la pièce réelle par l'acquisition $3 \mathrm{D}$ vision, qui permet également de définir le nombre de passes nécessaires. En ponçage, le système de compliance permet d'ajuster la trajectoire théorique au profil réel de la pièce (Fig. 6b). Le positionnement de la pièce dans l'espace de travail est fortement contraint par la taille et la morphologie des pièces, et dicté par l'accessibilité de l'effecteur aux différentes surfaces (Fig. 7), lui-même contraint par des limitations articulaires supplémentaires liées à l'accastillage, à l'encombrement des trois têtes et au montage de bridage. Ceci nécessite une phase importante de simulation et de contrôle de collision.

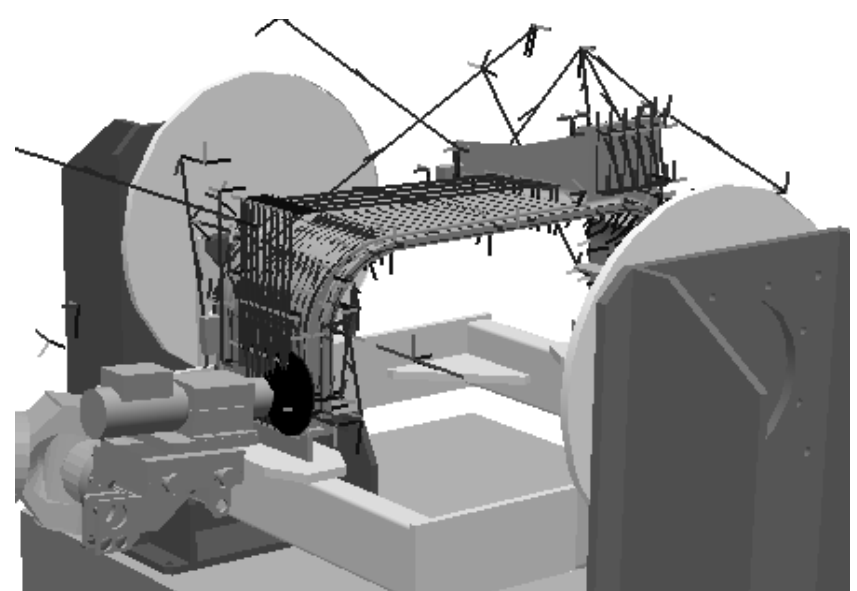

Fig. 7. Visualisation des trajectoires de ponçage et d'accessibilité aux surfaces dans RobotStudio ${ }^{\circledR}$.

\section{Critères d'optimisation}

L'optimisation des trajectoires consiste à placer la structure dans la configuration la plus favorable vis-àvis des procédés. Nous avons cherché à améliorer le comportement suivant deux axes : la précision et la capacité cinématique de la cellule [19].

\subsection{Critère de précision}

La diminution des imprécisions liées aux déformations de la structure nécessite l'étude de la rigidité de celle-ci et des sollicitations exercées.

\subsubsection{Rigidité de la structure}

$\mathrm{Au}$ cours des phases d'usinage et de ponçage, la structure est soumise à des efforts dus aux masses embarquées et aux phénomènes de coupe et d'abrasion. Une étude, s'appuyant sur un ensemble de travaux [16, 20], et complétée par une phase expérimentale, permet de conclure que la variation de la rigidité de la structure dépend de la configuration des jambes de la sous-structure parallèle ainsi que du dernier axe du poignet qui est mécaniquement le moins rigide. Nous cherchons donc à se placer dans la configuration qui minimise la sortie des jambes et le couple sur le dernier axe du poignet.

\subsubsection{Sollicitations sur la structure}

La structure est tout d'abord soumise aux efforts d'inertie et au poids de la charge embarquée. De part les vitesses imposées à l'effecteur, nous faisons ici l'hypothèse de négliger les effets de l'inertie sur la structure. Le robot est soumis au poids de l'ensemble broche-outil, de l'ordre de $45 \mathrm{~kg}$. La carte de rigidité de la base parallèle, construite expérimentalement, permet de définir 
un premier critère lié à la valeur articulaire moyenne des jambes de la sous-structure parallèle :

$$
c_{1}=\frac{q_{1}+q_{2}+q_{3}}{3}
$$

où $q_{1}, q_{2}, q_{3}$ sont les valeurs articulaires des jambes. En effet, la déformation induite par la sollicitation est relativement proportionnelle à la valeur moyenne de sortie des jambes. Plus cette valeur est faible et plus la rigidité de la sous-structure est grande. Le bras de levier lié au poids, appliqué au poignet étant faible, nous négligeons son influence sur le calcul du critère.

Dans notre configuration d'usinage, la résultante $\boldsymbol{F}_{x y}$ des efforts de coupe est orientée en moyenne à $110^{\circ}$ par rapport à la direction d'avance $\boldsymbol{d}$. Nous cherchons à minimiser le couple induit par cette composante sur le dernier axe du poignet de valeur articulaire $q_{6}$. Nous définissons un deuxième critère par :

$$
c_{2}=\left\|u_{F x y} \wedge u_{6}\right\|
$$

où $\boldsymbol{u}_{F x y}$ est vecteur directeur unitaire de la résultante des efforts de coupe, et $\boldsymbol{u}_{6}$ le vecteur directeur du dernier axe du poignet de valeur articulaire $q_{6}$.

\subsection{Critère cinématique}

L'étude cinématique a pour but d'exprimer un critère de performance lié à l'amélioration du comportement cinématique. On trouve plusieurs critères de performance cinématique qui caractérisent la dextérité du manipulateur [18], c'est-à-dire sa capacité à se déplacer et engendrer une vitesse à partir de sa position courante. Ils sont généralement définis à partir de la matrice jacobienne $\boldsymbol{J}$ du robot. On retrouve en particulier l'indice de manipulabilité en vitesse $w$, l'indice d'isotropie, donné par le conditionnement $K$ de la matrice jacobienne. L'inconvénient majeur de ces critères est qu'ils ne fournissent aucune indication de performance associée à une direction de déplacement. Dans notre cas, cette indication est importante puisque l'on cherche à obtenir un comportement volontairement non isotrope : une grande capacité en vitesse de l'effecteur selon la direction d'avance $\boldsymbol{d}$. La dualité vitesse/force donne alors un comportement peu rigide selon la direction d'avance et plus rigide suivant les autres directions. L'ellipsoïde de manipulabilité doit donc avoir son grand axe orienté suivant la direction d'avance $\boldsymbol{d}$. On utilise alors un premier critère de capacité en vitesse selon une direction $\boldsymbol{d}$, ratio de vitesse de l'effecteur :

$$
c_{3}=\boldsymbol{u}_{\boldsymbol{d}}^{T}\left(\boldsymbol{J} \boldsymbol{J}^{T}\right)^{-1} \boldsymbol{u}_{\boldsymbol{d}}
$$

où $\boldsymbol{u}_{\boldsymbol{d}}$ représente un vecteur unitaire de vitesse dans la direction $\boldsymbol{d}$ et $\boldsymbol{J}$ la matrice jacobienne. Ainsi, la diminution de ce critère traduit une augmentation de la capacité en vitesse dans la direction $\boldsymbol{d}$. Cette marge permet de limiter les sollicitations sur les différents axes pilotés, et peut être utilisée ensuite pour l'amélioration de la productivité par l'optimisation des paramètres des deux procédés.

\section{Optimisation des trajectoires}

\subsection{Méthode de résolution}

La résolution du problème d'optimisation consiste à déterminer pour chaque pose de la trajectoire chacun des trois paramètres suivants : valeurs articulaires du track $q_{7}$, du retourneur $q_{8}$, et l'angle de réorientation $\theta$ autour de l'axe outil.

Avec trois critères d'optimisation $\left(c_{1}, c_{2}, c_{3}\right)$, nous sommes dans le cas d'une optimisation multi-objectifs. L'utilisation d'une méthode d'agrégation des objectifs permet d'éviter la construction d'un front de Pareto nécessitant un choix entre solutions non dominées. Pour la résolution de la redondance [21], une méthode classique est l'utilisation de la projection sur le noyau de la matrice jacobienne :

$$
\dot{q}=\boldsymbol{J}^{+} \dot{x}+\underbrace{\left(\boldsymbol{I}-\boldsymbol{J}^{+} \boldsymbol{J}\right) z}_{\boldsymbol{J}_{h}}
$$

où $\boldsymbol{J}^{+}=\boldsymbol{J}^{T}\left(\boldsymbol{J} \boldsymbol{J}^{T}\right)^{-1}$ désigne la pseudo inverse de $\boldsymbol{J}, \boldsymbol{z}$ est un vecteur de même dimension que $q, \boldsymbol{I}$ est la matrice identité (de la dimension de $q$ ) et $\boldsymbol{J}_{h}$ la matrice de projection de $\boldsymbol{z}$ sur le noyau de $\boldsymbol{J}$. Le vecteur $\boldsymbol{z}$ est dans notre cas défini comme le gradient d'une fonction objectif $C$ construite par agrégation des objectifs initiaux. Une des difficultés des méthodes d'agrégation réside dans le choix du poids affecté à chaque critère. Pour modifier l'importance relative de chaque critère suivant la nécessité, nous utilisons une méthode à poids variables [22]. La forme de la fonction objectif $C$ devient alors :

$$
C(q)=\sum_{i=1}^{k} w_{i}\left(\bar{c}_{i}(x)\right) \bar{c}_{i}(q) \text { avec } \bar{c}_{i}(q)=\frac{c_{i}(q)-c_{i \min }}{c_{i \max }-c_{i \min }}
$$

où $w_{i}\left(\bar{c}_{i}(q)\right)$ est la fonction de poids dépendant du critère $\bar{c}_{i}$ variant entre 0 et 1 .

\subsection{Contraintes articulaires}

Cette méthode d'agrégation permet d'intégrer facilement les contraintes du robot, en particulier les butées articulaires, sous forme de critères supplémentaires. On utilise alors un critère classique d'éloignement des butées articulaires exprimé sous la forme :

$$
c_{4}=\frac{1}{6} \sum_{i=1}^{n}\left(\frac{q_{i}-q_{i \mathrm{moy}}}{q_{i \mathrm{moy}}-q_{i M}}\right)^{2}
$$

où $q_{i \text { moy }}$ représente la valeur moyenne de l'articulation $q_{i}$ et $q_{i M}$ sa valeur maximale. L'utilisation de seuils appropriés, associés à ce critère, permet d'éviter le risque classique de ramener toutes les articulations à leur valeur moyenne. Ils permettent alors de ne prendre en compte ce critère qu'à proximité des butées articulaires. Un critère du même type est utilisé pour l'éloignement de la singularité du poignet. 


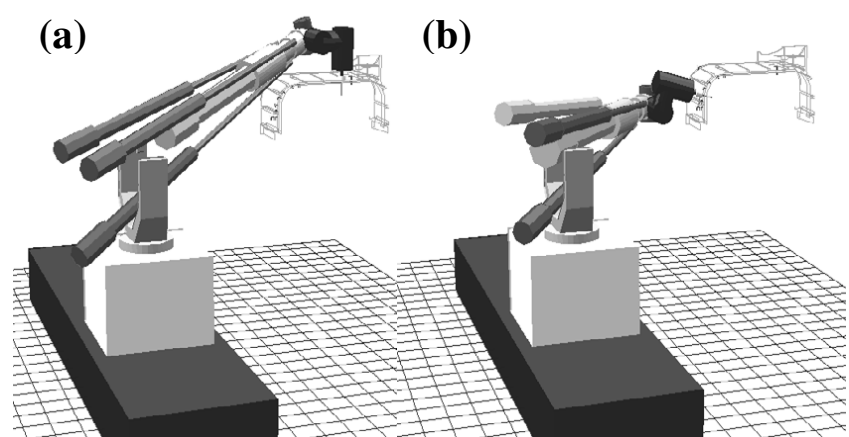

(c)

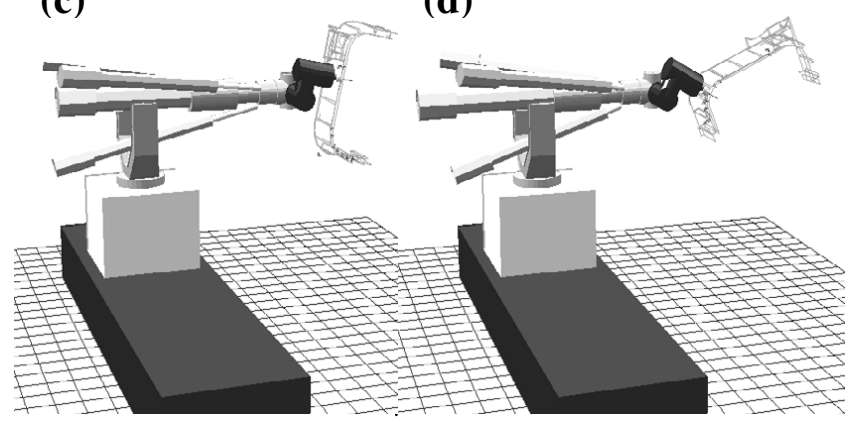

Fig. 8. Simulation sous Matlab ${ }^{\circledR}$ avant optimisation où apparaissent en foncé les jambes hors courses $(a, b)$, et après optimisation (c, d).

\subsection{Simulation et résultats}

La simulation de la cellule et les fonctions d'optimisation sont implémentées sous Matlab ${ }^{\circledR}$. Les méthodes décrites permettent de déterminer les 3 paramètres $(\theta$, $\left.q_{7}, q_{8}\right)$ associés à chaque pose et ainsi de définir un comportement optimal le long de la trajectoire [23].

Nous présentons (Fig. 9) l'évolution des critères $c_{1}$, $c_{2}$ et $c_{3}$ au cours d'une trajectoire (Fig. $6 \mathrm{~b}$ ) réglée pour les conditions d'usinage. La redéfinition des valeurs $q_{7}$ et $q_{8}$ montrent une diminution forte du critère $c_{1}$ (Fig. 9b) améliorant la précision et celle de l'angle $\theta$ une diminution du critère $c_{2}$ limitant les sollicitions sur le dernier axe du poignet (Fig. 9c).

Les résultats de l'optimisation ont été testés et appliqués sur un ensemble de pièces industrielles (Fig. 10). Le comportement obtenu permet de minimiser les sollicitations de la structure et de satisfaire les contraintes des procédés, avec une tolérance affleurement en usinage inférieure à $0,2 \mathrm{~mm}$ sur l'ensemble des plots et une bonne qualité de ponçage.

\section{Conclusion}

Cet article présente la démarche de mise en œuvre d'une cellule robotisée innovante, dans un contexte de haute technologie industrielle, pour le parachèvement des pièces de fonderie de grandes dimensions. Les contraintes prépondérantes sont l'accessibilité, la vitesse d'avance de

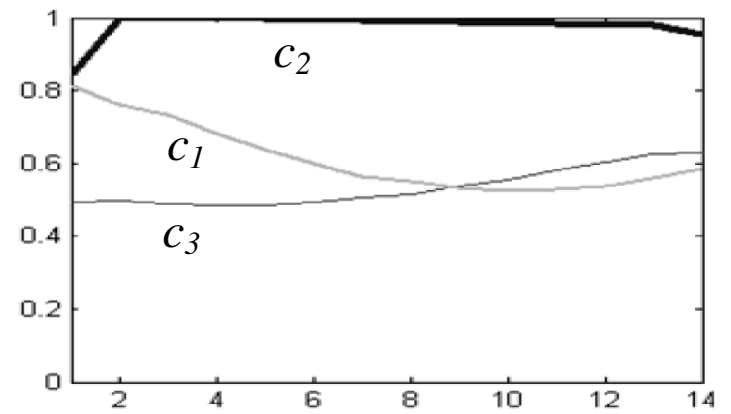

(a)

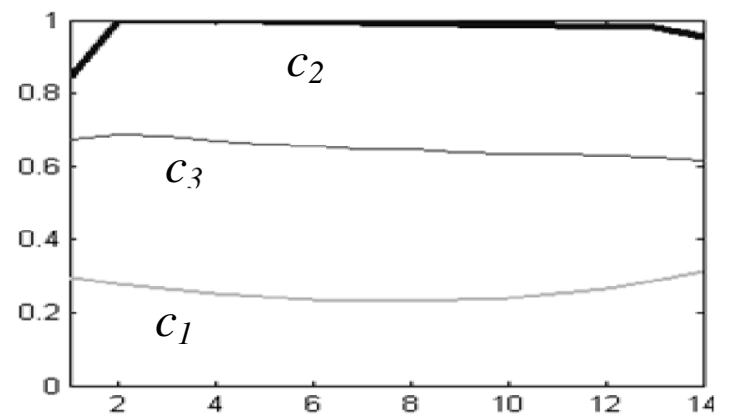

(b)

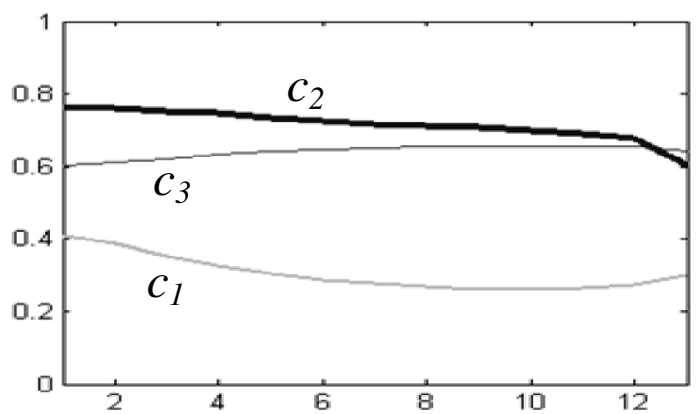

(c)

Fig. 9. Évolution des critères sans optimisation (a), après optimisation sans réorientation $(b)$, après optimisation et réorientation de $\theta(\mathrm{c})$.

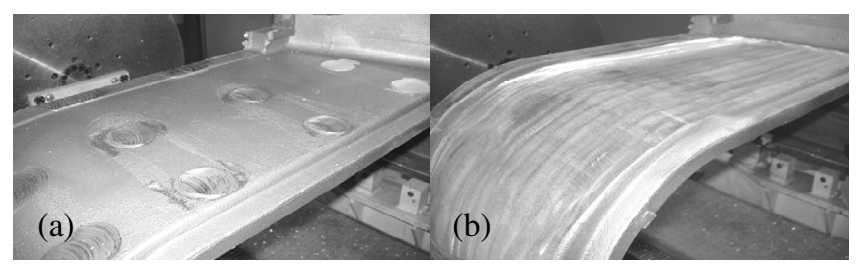

Fig. 10. Résultat après usinage des plots (a) et après ponçage (b).

l'effecteur et la précision qui nécessite une phase d'optimisation du comportement. Les résultats de simulation ont été testés et appliqués sur des pièces industrielles. Les trajectoires obtenues et validées permettent ainsi de satisfaire les spécifications attendues sur la pièce et les contraintes des procédés. Les résultats obtenus sont conformes aux exigences industrielles en termes de qualité de réalisation, programmation et automatisation du parachèvement. 
Remerciements. Ces travaux ont été réalisés dans le cadre du projet Eureka SANDCAST soutenu par la communauté Européenne (Eureka No. 02490 6090).

\section{Références}

[1] S.-C. Lin, M.-L. Wu, A study of the effects of polishing parameters on material removal rate and non-uniformity, Machine tools Manuf. 42 (2002) 99-103

[2] A. Roswell, F. Xi, G. Liu, Modelling and analysis of contact stress for automated polishing, Machine Tools Manuf. 46 (2006) 424-435

[3] H. Tam, O. Lui, A. Mok, Robotic polishing of free-form surfaces using scanning paths, Mater. Proc. Technol. 95 (1999) 191-200

[4] L. Feng-yun, L. Tian-sheng, Development of a robot system for complex surfaces polishing based CL data, Adv. Manuf. technol. 26 (2005) 1132-1137

[5] X. Pessoles, C. Tournier, Automatic polishing process of plastic injection molds on a 5-axis milling center, Mater. Proc. Technol. 209 (2009) 3665-3673

[6] J.P. Merlet, Les robots parallèles, Hermès, Paris, 1997

[7] 0. Company, Machines outils rapides à structure parallèle, Méthodologie de conception, applications et nouveaux concepts, Thèse, Univ. Montpellier II, 2000

[8] M. Geldart, P. Webb, H. Larsson, M. Backstrom, N. Gindy, K. Rask, A direct comparison of the machining performance of a varix 5 axis parallel kinematic machining centre with conventional 3 and 5 axis machine tools, Machine Tools Manuf. 43 (2003) 1107-1116

[9] M. Terrier, Optimisation du processus de fabrication en usinage à grande vitesse sur machines-outils à structure parallèle, Thèse, École Centrale de Nantes, 2005

[10] M.-C. Lee, S.J. Go, J.Y. Jung, M.H. Lee, Polishing robot attached to a machining center for a freely-curved surface die, Korean Society of Precision Eng. 3 (2002) 5-104

[11] C. Zielinski, W. Szynkiewicz, K. Mianowski, K. Nazarczuk, Mechatronic design of open-structure multirobot controllers, Mechatronics 11 (2001) 987-1000
[12] S. Bissey, Développement d'un modèle d'efforts de coupe applicable à des familles d'outils, Ph.D. Thesis, Arts et Métiers ParisTech, 2005

[13] Y. Quinsat, L. Sabourin, Optimal selection of machining direction for 3 axis milling of sculptured parts, J. Adv. Manuf. Technol. 27 (2006) 1132-1139

[14] L. Liao, F. Xi, K. Liu, Modeling and control of automated polishing/deburring process using a dual-purpose compliant toolhead, Machine tools Manuf. 48 (2008) 14541463

[15] E. Brinksmeier, O. Riemer, A. Gessenharter, Finishing of structured surfaces by abrasive polishing, Precision Eng. 30 (2006) 325-336

[16] H. Chanal, E. Duc, P. Ray, A study of the impact of machine tool structure on a machining process, Machine Tools Manuf. 46 (2006) 98-106

[17] G. Pritschow, C. Eppler, T. Garber, Influence of the dynamic stiffnes on the accuracy of PKM, (éd.), 3rd Chemnitz parallel Kinematics seminar, Chemnitz, Germany, 2002, pp. 313-333

[18] E. Dombre, Analyse et modélisation des robots manipulateurs, Hermès, Paris, 2001

[19] V. Robin, Contribution à la mise en œuvre et l'optimisation d'une cellule robotisée : application au parachèvement des pièces de fonderie, Thèse, Univ. Blaise Pascal, 2007

[20] Y. Wang, T. Huang, X. Zhao, J.P. Mie \& al, Finite element analysis and comparison of two hybrid robots the Tricept and the TriVariant, (éd.), Intelligent Robots and Systems, Proceedings of the IEEE/RSJ, Beijing, China, 2006, pp. 490-495

[21] K.C. Park, P.H. Chang, S. Lee, Analysis and control of redundant manipulator dynamics based on an extended operational space, Robotica 19 (2001) 649-662

[22] K.K. Lee, M. Buss, Redondancy resolution with multiple criteria, (éd.), Intelligent Robots and Systems, Proceedings of the IEEE/RSJ, Beijing, China, 2006, pp. 598-603

[23] V. Robin, L. Sabourin, G. Gogu, Optimization of a robotized cell with redundant architecture, Robotics and Computer-Integrated Manufacturing 27 (2010) 13-21 